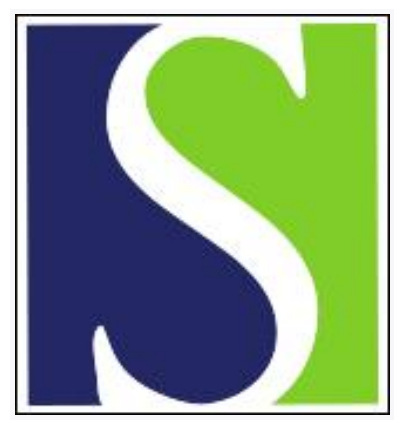

Scand J Work Environ Health 2020;46(4):417-428

https://doi.org/10.5271/sjweh.3878

Published online: 23 Jan 2020, Issue date: 01 Jul 2020

Occupational chemical exposures in pregnancy and fetal growth: evidence from the Born in Bradford Study

by Shirangi A, Wright J, Blair EM, McEachan RRC, Nieuwenhuijsen MJ

This is the first study to show that the risk of inadequate fetal growth as measured by percentage of optimal birth weight (POBW) $<85$ was elevated following occupational exposures to pesticides and phthalates. Further larger studies are needed to confirm this. Employers should provide workers with adequate education about potential chemical hazards in the workplace to ensure the complete protection of all workers' reproductive health.

Affiliation: Bradford Institute for Health Research, Bradford Teaching Hospitals, NHS Foundation Trust, Bradford UK BD9 6RJ. a.shirangi@murdoch.edu.au

Refers to the following texts of the Journal: 2006;32(1):61-66 2005;31(3):212-217

The following article refers to this text: 2020;46(4):335-338

Key terms: Born in Bradford Study; chemical exposure; EDC; endrocrine disrupting chemical; epidemiology; exposure; fetal growth; maternal characteristic; occupational chemical exposure; percentage of optimal birth weight; pregnancy; prospective cohort; still birth

This article in PubMed: www.ncbi.nlm.nih.gov/pubmed/31970422

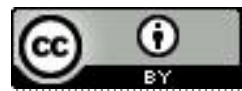




\title{
Occupational chemical exposures in pregnancy and fetal growth: evidence from the Born in Bradford Study
}

by Adeleh Shirangi, PhD, ${ }^{1-4}$ John Wright, FRCP, ${ }^{1}$ Eve M Blair, PhD, ${ }^{4}$ Rosemary RC McEachan, PhD, ${ }^{1}$ Mark J Nieuwenhuijsen, PhD, ${ }^{5-7}$

\begin{abstract}
Shirangi A, Wright J, Blair EM, McEachan RRC, Nieuwenhuijsen M. Occupational chemical exposures in pregnancy and fetal growth: evidence from the Born in Bradford Study. Scand J Work Environ Health. 2020;46(4):417-428. doi:10.5271/ sjweh.3878
\end{abstract}

Objectives This prospective birth cohort study evaluated the effect of occupational exposure to endocrine disrupting chemicals (EDC) during pregnancy on inadequate fetal growth as measured by small-for-gestational age (SGA) and inadequate fetal growth measured by percentage of optimal birth weight (POBW). The study also identified the maternal characteristics associated with an increased risk of exposure to EDC.

Methods We studied 4142 pregnant women who were in paid employment during pregnancy and participated in a population-based, prospective 2007-2011 birth cohort study, the Born in Bradford Study, with an estimated participation of $80 \%$. Job titles were coded at 26-28 weeks' gestation at a 4-digit level according to 353 unit groups in the 2000 UK Standard Occupational Classification. They were then linked to expert judgment on exposure to each of ten EDC groups as assessed through a job exposure matrix (JEM). We performed generalized estimation equation modelling by a modified Poisson regression to assess the risk of POBW and SGA associated with an increased risk of chemical exposures.

Results The frequency of POBW $<85$ significantly increased for mothers exposed to pesticides [adjusted risk ratio $\left(\mathrm{RR}_{\mathrm{adj}}\right) 3.72,95 \%$ confidence interval $\left.(\mathrm{CI}) 1.40-9.91\right]$ and phthalates $\left(\mathrm{RR}_{\mathrm{adj}} 3.71,95 \% \mathrm{CI} 1.62-8.51\right)$. There was a 5-fold increase risk of SGA for mothers exposed to pesticides $\left(\mathrm{RR}_{\mathrm{adj}} 5.45,95 \% \mathrm{CI} 1.59-18.62\right)$. Veterinary nurses and horticultural trades were most frequently associated with exposure to pesticides while hairdressers, beauticians, and printing machine minders were associated with phthalates.

Conclusion Maternal occupational exposure to estimated concentrations of pesticides and phthalates is associated with impaired fetal growth.

Key terms EDC; endrocrine disrupting chemical; epidemiology; maternal characteristic; percentage of optimal birth weight; prospective cohort; still birth.

Endocrine disrupting chemicals (EDC) are exogenous human-made substances that alter hormone regulation through interference with the endocrine system (1). They include many classes of chemicals such as pesticides, phthalates, polycyclic aromatic hydrocarbons (PAH), alkyl phenolic compounds (ALP), solvents, cytotoxic drugs, and anaesthetic gases. Global concerns have been raised in recent years over the potential adverse health effects of exposure to EDC (1-3). The endocrine system regulates many essential body functions such as growth, behavior, and reproduction through the controlled release of hormones $(1,4)$. The most sensitive windows of exposure to EDC are during fetal development and puberty (1). With an increasing number of women active in the labor force in both developed and developing countries, many will work during their reproductive years $(5,6)$ and likely be exposed to a variety of chemicals during pregnancy. Associations between prenatal exposure to EDC and a num-

1 Bradford Institute for Health Research, Bradford Teaching Hospitals, NHS Foundation Trust, Bradford UK.

2 College of Arts, Business, Law, and Social Sciences, Murdoch University, Perth, WA, Australia.

3 School of Population \& Global Health, the University of Western Australia, Perth, WA, Australia.

4 Telethon Kids Institute, Perth, WA, Australia.

5 ISGlobal, Barcelona, Spain.

6 Universitat Pompeu Fabra (UPF), Barcelona, Spain.

7 CIBER Epidemiologíay Salud Pública (CIBERESP), Barcelona, Spain.

Correspondence to: Dr Adeleh Shirangi, Bradford Institute for Health Research, Bradford Teaching Hospitals, NHS Foundation Trust, Bradford UK BD9 6RJ. [E-mail: a.shirangi@murdoch.edu.au] 
ber of adverse pregnancy outcomes have been reported, including miscarriage (7), birth defects (8-12), stillbirth (13), small-for-gestational age (SGA) (14), impaired fetal growth $(15,16)$, low birthweight (LBW) (17), and preterm birth (PTB) (18). However, there are limited prospective birth cohort studies to evaluate this association and despite these investigations, evidence of such effects in humans is inconclusive, and many EDC have not yet been evaluated in epidemiological research (5).

Babies born with inadequate fetal growth are at increased risk of life-threatening health problems, as well as long-term complications and developmental delays (19-23). Inadequate fetal growth is an important predictor of perinatal morbidity and mortality, a potential risk factor for cognitive disability later in childhood and coronary heart disease and hypertension in adult life (17-21). Despite extensive research, the causes of these adverse birth outcomes are incompletely understood but factors such as sociodemographic and socioeconomic status; lifestyle; reproductive history; medical conditions, such as diabetes and hypertension during pregnancy; as well as occupational and environmental exposures may be relevant (24-27). Their association with several workrelated risk factors is well established and has resulted in legislation, for example, considering exposure to specific chemicals, such as photoresistant solvents in the semiconductor industry or antineoplastic (cytotoxic) drugs in healthcare organizations, which have been declined over the past 20 years $(11,28)$. However, the scientific evidence is less consistent for many other EDC.

The primary objective of this study was to assess the effects of occupational exposures to estimated concentrations of EDC on the risk of SGA and inadequate fetal growth. The secondary objective was to identify the maternal characteristics associated with an increased risk of exposure to EDC.

\section{Methods}

The Born in Bradford Study is a population-based, prospective, longitudinal, and multi-ethnic birth cohort study that recruited 12453 pregnant women with 13959 pregnancies during 2007-2011. With an estimated participation rate of $80 \%$, the study monitors participants, their partners and off-spring until adulthood. Full details of the study methodology have been previously reported elsewhere $(29,30)$.

\section{Study design}

Figure 1 shows the selection of the study cohort. Information about job description and working conditions was collected primarily through a mid-pregnancy question-

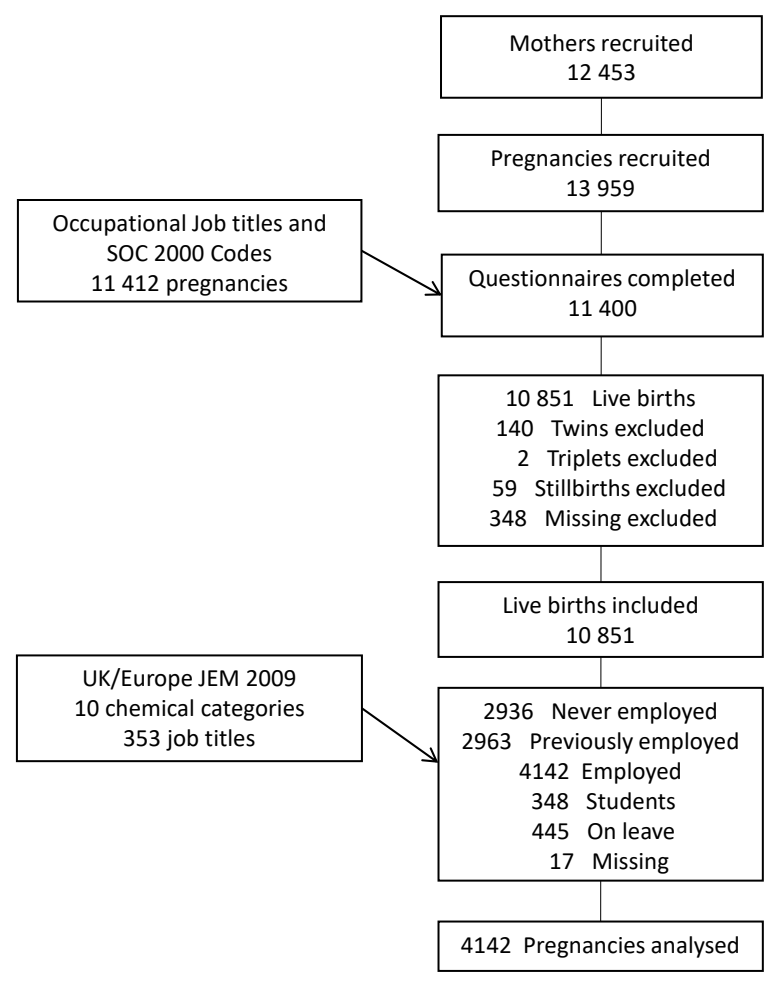

Figure 1. Flowchart of the steps in the selection of the study cohort.

naire at about 26-28 weeks' gestation. The questionnaire data was linked to maternity data and employment status for 11400 pregnancies. We selected women who gave birth to a live-born singleton, were in paid employment during pregnancy, and enrolled in the Born in Bradford Study prenatally. Of 11400 pregnancies, we excluded those with twins $(\mathrm{N}=140)$, triplets $(\mathrm{N}=2)$, stillbirths $(\mathrm{N}=59)$, and missing information on pregnancy outcome $(\mathrm{N}=348)$. Of the 10851 remaining, we excluded pregnancies where the mother was: not employed during pregnancy ( $\mathrm{N}=2963)$, Of the 10851 remaining, we excluded pregnancies where the mother was: not employed during pregnancy $(\mathrm{N}=2963)$, never employed $(\mathrm{N}=2936)$, a student $(\mathrm{N}=348)$, on sick leave $(\mathrm{N}=445)$, and missing information on the working situation $(\mathrm{N}=17)$. Therefore, $4142(38 \%)$ of mothers in paid employment during their pregnancies were eligible for analysis.

The Bradford Research Ethics Committee provided ethics approval for the study (reference 06/Q1202/48).

\section{Working condition and occupational coding}

Information concerning job title, type of business, selfemployment, and the four main tasks performed at work were used to classify the jobs according to the UK Classification of Occupations (31). We coded the job titles at a 4-digit level according to 353 unit groups in the 2000 
UK Standard Occupational Classification (31). The job titles were coded and validated through the ComputerAssisted Structure Coding Tool (Cascot) (32). The coded job titles subsequently linked to an updated UK job exposure matrix (JEM) for chemical exposure developed over the same period as this cohort study $(33,34)$.

\section{Exposure assessment}

In 2009, Brouwers et al (34) developed this JEM, which considers the 353 job titles, adapted from the van Tongeren JEM of 2002 (33). Three occupational hygienists estimated the job-specific risk of exposure to each of ten chemicals groups: $\mathrm{PAH}$, polychlorinated organic compounds, pesticides, phthalates, organic solvents, bisphenol A, ALP, brominated flame-retardants, metals, and a miscellaneous group: as unlikely (score $=0$ ), possible $($ score $=1$ ) or probable (score $=2$ ). In addition, broad and non-specific job titles were considered 'unclassifiable'. For this study, we collated the last two categories (possible and probable) into one indicating the occurrence of exposure to EDC was more likely than unlikely. No distinction was made between the various routes of exposure (inhalation, ingestion, or dermal). For many chemicals, most of the population experiences some level of exposure through diet or widely used consumer products. The JEM exposure score refers to the probability that the occupational exposure exceeds this background level.

\section{Measures of birth outcomes}

Information about gestational age, gender, weight, length and head circumference at birth was obtained from medical records and hospital registries to allow the following variables to be created.

Gestational age was based on the actual and estimated date of delivery calculated by the physician or midwife from the dating scan (if available) or last menstrual period.

$S G A$ was defined as a birth weight less than the 10th customized centile, using GROW software from 2013 (35, 36), www.gestation.net/cc/about.htm (37). The calculation of SGA was derived from maternal characteristics, birth weight and gestational age data recorded in the electronic maternity system at the Bradford Research Institute.

Optimal birthweight was estimated for each birth using a model derived from a population of singletons not exposed to any of the common risk factors for growth anomaly, with terms for infant gender, gestational duration, and maternal height and parity by a method validated and corrected for births before 30 weeks' gestation $(38,39)$. Appropriateness of intrauterine growth is inferred from the ratio of the observed-to-optimal birth weight expressed as a percentage, percentage of optimal birth weight (POBW). The 10th percentile of weight in the original population was a POBW of $87 \%$ (38), therefore our criterion for inadequate fetal growth of POBW of $<85$ represents a slightly more stringent criterion than the 10th percentile, the criterion used for SGA. The method has been used in previously published studies $(23,40,41)$.

\section{Maternal characteristics / confounder assessment}

The following potentially confounding factors were also solicited with the mid-term questionnaire: mother's sociodemographic, lifestyle, ethnicity, medical, and socioeconomic status [index of multiple deprivation 2010 (IMD)] as described in table 1. These characteristics are considered potential confounders for both aims of study investigation. We also considered each group of EDC as independent variables in the analysis to address the first aim of this study. Chemical exposures with numbers fewer than five records, which include polychlorinated organic compounds, bisphenol A, and flame-retardants, were not included in the analysis. As such, seven groups of chemicals were included in the analysis.

\section{Strategy of statistical analysis}

We used univariate and multivariate analyses with risk ratios (RR) and 95\% CI (CI) generated using generalized estimation equation (GEE) modelling by a modified Poisson regression, with robust error variance (42, 43). Findings at $\mathrm{P}<0.05$ were considered significant. The two crude and adjusted models estimated the risk of dependent variables with independent variables as shown in tables 2 and 3. All independent variables were categorical. For example, in table 2, all co-variables were screened by cross-tabulations, $\mathrm{Chi}^{2}$ test and also the Mantel-Haenzel adjusted odds ratio (OR) with separate SGA and POBW variables. If significant at $\mathrm{P}<0.2$, the co-variables were entered into fully adjusted multivariate models for both SGA and POBW. Backwardsstepwise regression was used to simplify the models by sequentially removing non-significant variables that did not reduce how well the data fitted the models. Covariates were included in the multivariate model if the difference between the crude and adjusted RR was $>10 \%$ for either outcome measure. For reasons of comparison and based on evidence from previous literature, maternal age, education, alcohol consumption, and job hours were included by default, independent of statistical significance. Interaction effects were examined for statistical significance. The analysis of $\mathrm{POBW}<85$ was also stratified by ethnicity. All preceding calculations were made using the statistical program STATA (StataCorp, College Station, TX, USA). 
Table 1. Characteristics of 4142 pregnant women enrolled in Born in Bradford Study and their associations with crude risk of impaired fetal growth[BMI=body mass index; $E D C=$ endrocrine disrupting chemical; $G C S E=$ general certificate of secondary education; IMD=index of multiple deprivation for Bradford; $\mathrm{PAH}=$ polycyclic aromatic hydrocarbons; $\mathrm{POBW}=$ percentage of optimal birth weight; $\mathrm{SGA}=$ small-for-gestational age.]

\begin{tabular}{|c|c|c|c|c|c|c|}
\hline Characteristics & Number ${ }^{a}$ & $\%$ & SGA & Crude SGA risk & $\mathrm{POBW}<85$ & Crude $\mathrm{POBW}<85$ risk \\
\hline Total & 4142 & 100 & 451 & 0.109 & 740 & 0.179 \\
\hline \multicolumn{7}{|l|}{ Ethnic origin } \\
\hline British white & 2482 & 59.92 & 188 & 0.076 & 323 & 0.135 \\
\hline South Asian & 1188 & 28.68 & 228 & 0.191 & 352 & 0.307 \\
\hline Other & 472 & 11.4 & 35 & 0.074 & 65 & 0.145 \\
\hline \multicolumn{7}{|l|}{ Age (years) } \\
\hline$\leq 35$ & 3683 & 88.92 & 482 & 0.109 & 659 & 0.185 \\
\hline$>35$ & 459 & 11.88 & 49 & 0.107 & 81 & 0.183 \\
\hline \multicolumn{7}{|l|}{ Education (mother) } \\
\hline$<5$ GCSE equivalents & 315 & 7.62 & 29 & 0.092 & 70 & 0.235 \\
\hline 5 GCSE equivalents & 1120 & 27.11 & 142 & 0.126 & 210 & 0.195 \\
\hline A-level equivalent ${ }^{\mathrm{b}}$ & 819 & 19.82 & 86 & 0.105 & 148 & 0.184 \\
\hline$>$ A level & 1470 & 35.58 & 155 & 0.105 & 253 & 0.179 \\
\hline Other degrees & 333 & 8.06 & 31 & 0.093 & 44 & 0.137 \\
\hline Unknown & 75 & 1.82 & 8 & 0.107 & 14 & 0.192 \\
\hline \multicolumn{7}{|l|}{ Smoking (cigarettes) } \\
\hline 0 & 3492 & 84.37 & 354 & 0.101 & 575 & 0.171 \\
\hline 1-5 per day & 286 & 6.91 & 38 & 0.133 & 68 & 0.245 \\
\hline$>5$ per day & 361 & 8.72 & 59 & 0.164 & 97 & 0.275 \\
\hline \multicolumn{7}{|l|}{ Other tobacco products } \\
\hline No & 4105 & 99.32 & 445 & 0.108 & 730 & 0.184 \\
\hline Yes & 28 & 0.68 & 5 & 0.178 & 8 & 0.296 \\
\hline \multicolumn{7}{|l|}{ Drugs during pregnancy } \\
\hline No & 3528 & 99.07 & 379 & 0.107 & 633 & 0.183 \\
\hline Yes & 33 & 0.93 & 7 & 0.212 & 11 & 0.354 \\
\hline \multicolumn{7}{|l|}{ Alcohol } \\
\hline No /occasionally & 2981 & 72.09 & 352 & 0.118 & 581 & 0.202 \\
\hline Yes & 1154 & 27.91 & 98 & 0.085 & 158 & 0.142 \\
\hline \multicolumn{7}{|l|}{ Vitamin/iron } \\
\hline No & 2465 & 59.58 & 284 & 0.115 & 457 & 0.193 \\
\hline Yes & 1672 & 40.42 & 166 & 0.099 & 282 & 0.173 \\
\hline \multicolumn{7}{|l|}{ Body mass index } \\
\hline Normal & 1087 & 29.99 & 165 & 0.152 & 263 & 0.249 \\
\hline Overweight & 1606 & 39.88 & 166 & 0.103 & 268 & 0.174 \\
\hline Obese & 1328 & 32.98 & 100 & 0.075 & 171 & 0.134 \\
\hline Underweight & 6 & 0.15 & 3 & 0.5 & 4 & 0.666 \\
\hline \multicolumn{7}{|l|}{ Parity } \\
\hline First child & 2146 & 53.7 & 279 & 0.13 & 392 & 0.182 \\
\hline Second child \& higher & 1850 & 46.3 & 159 & 0.086 & 348 & 0.188 \\
\hline \multicolumn{7}{|l|}{ Baby sex } \\
\hline Female & 1973 & 47.63 & 231 & 0.117 & 377 & 0.197 \\
\hline Male & 2169 & 52.37 & 220 & 0.101 & 363 & 0.175 \\
\hline \multicolumn{7}{|l|}{ Age at first period (years) } \\
\hline $12-13$ & 1795 & 45.4 & 182 & 0.101 & 293 & 0.169 \\
\hline$<12$ & 598 & 15.12 & 73 & 0.122 & 116 & 0.201 \\
\hline$>13$ & 1561 & 39.48 & 176 & 0.113 & 294 & 0.195 \\
\hline Marital status & & & & & & \\
\hline Married/re-married & 2489 & 60.15 & 319 & 0.128 & 504 & 0.209 \\
\hline Single & 1537 & 37.14 & 128 & 0.0 .83 & 219 & 0.147 \\
\hline Separated/divorced/widowed & 112 & 2.71 & 4 & 0.036 & 17 & 0.165 \\
\hline IMD 2010 score & & & & & & \\
\hline 1 (most deprived) & 936 & 23.12 & 140 & 0.149 & 225 & 0.248 \\
\hline 2 & 946 & 23.37 & 118 & 0.125 & 190 & 0.207 \\
\hline 3 & 1010 & 24.95 & 92 & 0.091 & 164 & 0.17 \\
\hline 4 & 875 & 21.62 & 70 & 0.08 & 115 & 0.136 \\
\hline 5 (least deprived) & 281 & 6.94 & 19 & 0.067 & 32 & 0.118 \\
\hline Finance & & & & & & \\
\hline Better off & 1260 & 30.59 & 132 & 0.105 & 221 & 0.181 \\
\hline About the same & 2027 & 49.21 & 225 & 0.111 & 368 & 0.189 \\
\hline Worse off & 832 & 20.2 & 90 & 0.108 & 146 & 0.182 \\
\hline Job hours/week & & & & & & \\
\hline$\leq 35$ & 2204 & 53.37 & 257 & 0.116 & 428 & 0.202 \\
\hline$>35$ & 1926 & 46.63 & 193 & 0.1 & 310 & 0.166 \\
\hline Work type & & & & & & \\
\hline Most time/sitting & 1678 & 47.86 & 172 & 0.102 & 276 & 0.168 \\
\hline Most time/standing & 1387 & 39.56 & 172 & 0.124 & 280 & 0.205 \\
\hline Physical effort & 441 & 12.58 & 38 & 0.086 & 81 & 0.187 \\
\hline
\end{tabular}


Table 1. continued

\begin{tabular}{|c|c|c|c|c|c|c|}
\hline Characteristics & Number ${ }^{a}$ & $\%$ & SGA & Crude SGA risk & $\mathrm{POBW}<85$ & Crude POBW $<85$ risk \\
\hline \multicolumn{7}{|l|}{ Occupational group (Mother) } \\
\hline Managers/seniors & 320 & 7.74 & 29 & 0.09 & 45 & 0.146 \\
\hline Professionals & 439 & 10.62 & 43 & 0.098 & 73 & 0.173 \\
\hline Associated professionals & 676 & 16.35 & 63 & 0.093 & 100 & 0.153 \\
\hline Administration/secretarial & 717 & 17.34 & 89 & 0.124 & 139 & 0.2 \\
\hline Skilled trades & 45 & 10.09 & 4 & 0.089 & 8 & 0.182 \\
\hline Personal service & 815 & 19.71 & 83 & 0.101 & 140 & 0.178 \\
\hline Customer service & 500 & 12.72 & 66 & 0.123 & 97 & 0.186 \\
\hline Machine operatives & 53 & 1.35 & 7 & 0.127 & 14 & 0.269 \\
\hline Elementary occupation & 510 & 12.98 & 67 & 0.125 & 123 & 0.24 \\
\hline \multicolumn{7}{|l|}{ Gestational diabetes } \\
\hline No & 3878 & 93.78 & 434 & 0.112 & 782 & 0.188 \\
\hline Yes & 257 & 6.22 & 17 & 0.066 & 38 & 0.152 \\
\hline \multicolumn{7}{|l|}{ Preclampsia } \\
\hline No & 3844 & 97 & 407 & 0.106 & 675 & 0.181 \\
\hline Yes & 119 & 3 & 27 & 0.227 & 37 & 0.322 \\
\hline \multicolumn{7}{|l|}{ Pre-existing hypertension } \\
\hline No & 3926 & 98.82 & 427 & 0.108 & 702 & 0.185 \\
\hline Yes & 47 & 1.18 & 8 & 0.17 & 13 & 0.289 \\
\hline \multicolumn{7}{|l|}{ Any EDC exposure } \\
\hline No & 3777 & 92.66 & 417 & 0.11 & 673 & 0.184 \\
\hline Yes & 299 & 7.34 & 27 & 0.09 & 54 & 0.186 \\
\hline \multicolumn{7}{|l|}{ Exposure to PAH } \\
\hline No & 4012 & 98.43 & 438 & 0.109 & 716 & 0.185 \\
\hline Yes & 64 & 1.57 & 6 & 0.094 & 11 & 0.18 \\
\hline \multicolumn{7}{|l|}{ Exposure to pesticides } \\
\hline No & 4069 & 99.83 & 442 & 0.108 & 724 & 0.185 \\
\hline Yes & 7 & 0.17 & 2 & 0.285 & 3 & 0.429 \\
\hline \multicolumn{7}{|l|}{ Exposure to phthalates } \\
\hline No & 4000 & 98.14 & 440 & 0.11 & 717 & 0.185 \\
\hline Yes & 76 & 1.86 & 4 & 0.053 & 10 & 0.135 \\
\hline \multicolumn{7}{|l|}{ Exposure to organic solvents } \\
\hline No & 3895 & 95.56 & 425 & 0.109 & 690 & 0.184 \\
\hline Yes & 181 & 4.44 & 19 & 0.105 & 37 & 0.209 \\
\hline \multicolumn{7}{|l|}{ Exposure to akylphenolics } \\
\hline No & 3917 & 96.1 & 428 & 0.109 & 693 & 0.184 \\
\hline Yes & 159 & 3.9 & 16 & 0.101 & 34 & 0.219 \\
\hline \multicolumn{7}{|l|}{ Exposure to metals } \\
\hline No & 4028 & 98.82 & 441 & 0.109 & 722 & 0.186 \\
\hline Yes & 48 & 1.18 & 3 & 0.062 & 5 & 0.109 \\
\hline \multicolumn{7}{|l|}{ Exposure to miscellaneous } \\
\hline No & 4006 & 98.34 & 441 & 0.11 & 720 & 0.186 \\
\hline Yes & 70 & 1.72 & 3 & 0.043 & 7 & 0.103 \\
\hline
\end{tabular}

${ }^{a}$ Missing not included in the analysis.

${ }^{\mathrm{b}} \mathrm{A}$-Levels, A-C equates to Level 2 attainment defined by the 2011 revision of the International Standard Classification of Education; $\geq 2$ advanced levels or equivalent qualifications equate to Level 3 educational attainment.

\section{Results}

Table 1 describes the maternal characteristics of the 4142 eligible pregnancies and gives crude risks of SGA $(10.9 \%)$, and POBW $<85$ (17.9\%). The cohort was multiethnic: $\sim 60 \%$ were classified as Caucasian British, $\sim 29 \%$ South Asian (Pakistani/Bangladesh/India), and $\sim 11 \%$ other. The results from crude risks indicate that women were more likely to have babies with inadequate fetal growth as measured by both $\mathrm{SGA}$ and $\mathrm{POBW}<85$, if they are from a South Asian ethnicity group, less educated, smoke, use drugs, live in most derived areas, or have preeclampsia or pre-existing hypertension.

Table 2 presents the univariate and multivariate estimates of RR for each of the two outcomes associated with statistically significant risk factors. In the multivariate analyses, all maternal characteristics and each category of seven groups of chemicals (those with numbers more than five records) were included except vitamin/iron supplementation and financial status, which were not statistically significant. There were no significant differences between occupational groups for either outcome. However, work involving standing most of time was associated with a $25 \%$ increased risk of having a baby with inadequate fetal growth.

\section{Effects of EDC on the risk of SGA}

In multivariate analysis, the proportion of infants with SGA among women likely occupationally exposed to $\mathrm{PAH}$, pesticides, phthalates, or ALP was statistically non-significantly higher than among women in the refer- 
Table 2. Univariate and multivariate relative risk (RR) estimation using generalized estimation model by a modified Poisson regression of the effects of maternal occupational exposures to endocrine disrupter chemicals (EDC) on risk of inadequate fetal growth in infants born in Bradford. Bold denotes significance $(\mathrm{P}<0.05)$. [Cl=confidence interval; $\mathrm{BMI}=$ body mass index; $\mathrm{GCSE}=$ general certificate of secondary education; IMD=index of $\mathrm{mul}-$ tiple deprivation for Bradford; $\mathrm{PAH}=$ polycyclic aromatic hydrocarbons; POBW=percentage of optimal birth weight; $\mathrm{SGA}=$ small-for-gestational age.]

\begin{tabular}{|c|c|c|c|c|c|c|c|c|}
\hline \multirow[t]{2}{*}{ Characteristics } & \multirow{2}{*}{$\frac{\text { SGA }}{\text { Crude RR }(95 \% \mathrm{Cl})}$} & \multirow[t]{2}{*}{ P-value } & \multirow{2}{*}{$\begin{array}{c}\mathrm{SGA} \\
\mathrm{RR}_{\mathrm{adj}}{ }^{\mathrm{a}}(95 \% \mathrm{Cl})\end{array}$} & \multirow[t]{2}{*}{ P-value } & \multirow{2}{*}{$\begin{array}{c}\mathrm{POBW}<85 \\
\text { Crude RR }(95 \% \mathrm{Cl})\end{array}$} & \multirow[t]{2}{*}{ P-value } & \multirow{2}{*}{$\begin{array}{c}\mathrm{POBW}<85 \\
\mathrm{RR}_{\text {adj }}{ }^{\mathrm{a}}(95 \% \mathrm{Cl})\end{array}$} & \multirow[t]{2}{*}{ P-value } \\
\hline & & & & & & & & \\
\hline Exposed to $\mathrm{PAH}^{\mathrm{b}}$ & $0.86(0.40-1.84)$ & 0.69 & $1.25(0.53-2.94)$ & 0.60 & $0.97(0.57-1.67)$ & 0.92 & $0.91(0.46-1.80)$ & 0.79 \\
\hline Exposed to pesticides ${ }^{b}$ & $2.63(0.81-8.51)$ & 0.10 & $5.45(1.59-18.62)$ & 0.00 & $2.32(0.99-5.48)$ & 0.05 & $3.72(1.40-9.91)$ & 0.00 \\
\hline Exposed to phthalates ${ }^{b}$ & $0.48(0.18-1.24)$ & 0.13 & $1.69(0.34-8.41)$ & 0.52 & $0.73(0.41-1.29)$ & 0.28 & $3.71(1.62-8.51)$ & 0.00 \\
\hline Exposed to organic solvents ${ }^{b}$ & $0.96(0.62-1.48)$ & 0.86 & $1.08(0.36-3.29)$ & 0.88 & $1.13(0.85-1.52)$ & 0.39 & $0.89(0.44-1.80)$ & 0.74 \\
\hline Exposure to akylphenolics ${ }^{b}$ & $0.92(0.57-1.48)$ & 0.73 & $1.62(0.50-5.25)$ & 0.42 & $1.19(0.88-1.62)$ & 0.25 & $1.48(0.70-3.11)$ & 0.31 \\
\hline Exposure to metals ${ }^{b}$ & $0.57(0.19-1.71)$ & 0.31 & $0.58(0.15-2.16)$ & 0.42 & $0.58(0.25-1.34)$ & 0.2 & $0.49(0.16-1.49)$ & 0.21 \\
\hline Exposure to miscellaneous ${ }^{b}$ & $0.39(0.13-1.18)$ & 0.09 & $0.23(0.03-1.84)$ & 0.17 & $0.55(0.27-1.12)$ & 0.09 & $0.18(0.05-0.60)$ & 0.00 \\
\hline \multicolumn{9}{|l|}{ Occupational group (mother) } \\
\hline Managers/seniors & 1.00 & & 1.00 & & 1.00 & & 1.00 & \\
\hline Professionals & $1.08(0.69-1.69)$ & 0.72 & $0.92(0.50-1.69)$ & 0.80 & $1.00(0.81-1.24)$ & 0.96 & $1.18(0.75-1.86)$ & 0.47 \\
\hline Associated professionals & $1.02(0.68-1.56)$ & 0.86 & $1.10(0.64-1.91)$ & 0.72 & $1.01(0.76-1.45)$ & 0.91 & $1.35(0.89-2.04)$ & 0.15 \\
\hline Administration/secretarial & $1.36(0.92-2.03)$ & 0.12 & $1.18(0.69-2.01)$ & 0.54 & $1.21(1.00-1.46)$ & 0.04 & $1.49(0.98-2.24)$ & 0.06 \\
\hline Skilled trades & $0.98(0.36-2.66)$ & 0.97 & $0.63(0.18-2.20)$ & 0.48 & $1.07(0.68-1.66)$ & 0.76 & $0.99(0.41-2.37)$ & 0.99 \\
\hline Personal service & $1.12(0.75-1.68)$ & 0.57 & $1.07(0.61-1.87)$ & 0.81 & $1.15(0.95-1.39)$ & 0.13 & $1.19(0.78-1.82)$ & 0.41 \\
\hline Customer service & $1.36(0.89-2.05)$ & 0.14 & $1.05(0.60-1.83)$ & 0.85 & $1.15(0.94-1.40)$ & 0.15 & $1.18(0.77-1.81)$ & 0.44 \\
\hline Machine operatives & $1.40(0.65-3.05)$ & 0.39 & $0.64(0.22-1.86)$ & 0.41 & $1.51(1.09-2.09)$ & 0.01 & $1.42(0.66-3.06)$ & 0.37 \\
\hline Elementary occupation & $1.38(0.92-2.10)$ & 0.11 & $0.99(0.55-1.78)$ & 0.99 & $1.31(1.20-2.24)$ & 0.00 & $1.29(0.84-2.00)$ & 0.24 \\
\hline \multicolumn{9}{|l|}{ Work } \\
\hline Most time/sitting & 1.00 & & 1.00 & & 1.00 & & 1.00 & \\
\hline Most time/standing & $1.21(0.99-1.48)$ & 0.05 & $1.23(0.96-1.58)$ & 0.10 & $1.07(0.98-1.18)$ & 0.12 & $1.25(1.04-1.51)$ & 0.01 \\
\hline Physical effort & $0.84(0.60-1.17)$ & 0.31 & $0.85(0.57-1.28)$ & 0.44 & $0.97(0.83-1.12)$ & 0.66 & $1.19(0.91-1.56)$ & 0.18 \\
\hline \multicolumn{9}{|l|}{ Ethnic origin } \\
\hline British Caucasian & 1.00 & & 1.00 & & 1.00 & & 1.00 & \\
\hline South Asian & $2.53(2.11-3.03)$ & 0.00 & $2.69(1.94-3.73)$ & 0.00 & $1.97(1.81-2.13)$ & 0.00 & $2.43(1.91-3.09)$ & 0.00 \\
\hline Other & $0.97(0.69-1.38)$ & 0.90 & $0.86(0.52-1.41)$ & 0.56 & $1.02(0.87-1.19)$ & 0.77 & $1.06(0.76-1.47)$ & 0.72 \\
\hline \multicolumn{9}{|l|}{ Age (years) } \\
\hline$\leq 35$ & 1.00 & & 1.00 & & 1.00 & & 1.00 & \\
\hline$>35$ & $0.97(0.73-1.29)$ & 0.87 & $1.64(1.16-2.32)$ & 0.00 & $0.90(0.80-1.22)$ & 0.14 & $1.17(0.90-1.52)$ & 0.25 \\
\hline \multicolumn{9}{|l|}{ Education (mother) } \\
\hline$<5$ GCSE equivalents & 1.00 & & 1.00 & & 1.00 & & 1.00 & \\
\hline 5 GCSE equivalents & $1.37(0.94-2.00)$ & 0.10 & $1.51(0.95-2.41)$ & 0.08 & $0.84(0.72-0.97)$ & 0.02 & $0.96(0.73-1.28)$ & 0.82 \\
\hline A-level equivalent ${ }^{c}$ & $1.14(0.76-1.69)$ & 0.53 & $1.11(0.67-1.83)$ & 0.66 & $0.83(0.71-0.97)$ & 0.01 & $0.78(0.57-1.06)$ & 0.11 \\
\hline$>$ A level & $1.14(0.78-1.66)$ & 0.49 & $1.24(0.77-2.01)$ & 0.37 & $0.79(0.68-0.92)$ & 0.00 & $0.88(0.64-1.20)$ & 0.41 \\
\hline Other degrees & $1.00(0.62-1.63)$ & 0.97 & $1.29(0.71-2.33)$ & 0.41 & $0.69(0.56-0.85)$ & 0.00 & $0.70(0.45-1.09)$ & 0.11 \\
\hline Unknown/foreign & $1.15(0.55-2.42)$ & 0.70 & $1.26(0.51-3.12)$ & 0.61 & $0.71(0.49-1.02)$ & 0.06 & $0.91(0.50-1.67)$ & 0.78 \\
\hline \multicolumn{9}{|l|}{ Smoking (cigarettes) } \\
\hline 0 & 1.00 & & 1.00 & & 1.00 & & 1.00 & \\
\hline 1-5 per day & $1.31(0.96-1.79)$ & 0.09 & $1.63(1.13-2.35)$ & 0.00 & $1.20(1.04-1.39)$ & 0.01 & $1.62(1.26-2.09)$ & 0.00 \\
\hline$>5$ per day & $1.61(1.25-2.08)$ & 0.00 & $2.65(1.89-3.71)$ & 0.00 & $1.35(1.20-1.53)$ & 0.00 & $2.29(1.79-2.92)$ & 0.00 \\
\hline \multicolumn{9}{|l|}{ BMI } \\
\hline Normal & 1.00 & & 1.00 & & 1.00 & & 1.00 & \\
\hline Overweight & $0.68(0.55-0.83)$ & 0.00 & $0.76(0.60-0.97)$ & 0.02 & $0.75(0.68-0.82)$ & 0.00 & $0.76(0.64-0.91)$ & 0.00 \\
\hline Obese & $0.49(0.39-0.63)$ & 0.00 & $0.60(0.45-0.80)$ & 0.00 & $0.60(0.54-0.67)$ & 0.00 & $0.58(0.47-0.71)$ & 0.00 \\
\hline Underweight & $3.28(1.45-7.41)$ & 0.00 & $3.29(0.67-16.24)$ & 0.14 & $2.01(1.01-2.29)$ & 0.00 & $1.65(0.41-6.58)$ & 0.48 \\
\hline \multicolumn{9}{|l|}{ IMD 2010 score } \\
\hline 1 (most deprived) & 1.00 & & 1.00 & & 1.00 & & 1.00 & \\
\hline 2 & $0.83(0.66-1.04)$ & 0.11 & $0.82(0.62-1.07)$ & 0.15 & $0.88(0.79-0.98)$ & 0.02 & $0.77(0.64-0.94)$ & 0.01 \\
\hline 3 & $0.60(0.47-0.78)$ & 0.00 & $0.83(0.61-1.12)$ & 0.23 & $0.76(0.68-0.85)$ & 0.00 & $0.82(0.66-1.01)$ & 0.07 \\
\hline 4 & $0.53(0.41-0.70)$ & 0.00 & $0.81(0.58-1.15)$ & 0.25 & $0.71(0.63-0.80)$ & 0.00 & $0.78(0.60-0.99)$ & 0.04 \\
\hline 5 (least deprived) & $0.45(0.28-0.71)$ & 0.00 & $0.44(0.20-0.93)$ & 0.03 & $0.55(0.44-0.69)$ & 0.00 & $0.58(0.36-0.93)$ & 0.02 \\
\hline
\end{tabular}

${ }^{a}$ Adjusted for all variables in the table and job hours during pregnancy $(<35, \geq 35)$ : alcohol consumption (yes or no/occasionally), marital status (married/remarried, single, separated/divorced, widowed), parity (first child, second child \& higher), gestational diabetes (no, yes), preeclampsia (no, yes), pre-exciting hypertension (no, yes), other tobacco (no, yes), and drugs during pregnancy (no, yes).

b Unexposed=reference group (not shown in the table). Each group of EDC is included in the analysis as an independent variable.

${ }^{c}$ A-Level- A-C equates to Level 2 attainment defined by the 2011 revision of the International Standard Classification of Education; $\geq 2$ advanced Levels or equivalent qualifications equate to Level 3 educational attainment.

ence group, except for exposure to pesticides where it was 5 fold higher [adjusted RR (RRadj) $5.45,95 \% \mathrm{CI}$ 1.59-18.62]. No association was found between SGA and exposures to solvents, metals, and miscellaneous chemicals.
Effects of EDC on the risk of $P O B W<85$.

In multivariate analysis, the proportion of infants with POBW $<85$ among women likely occupationally exposed to pesticides (RRadj 3.72, 95\% CI 1.40-9.91) and phthalates 3-fold (RRadj 3.71, 95\% CI 1.62-8.51) was higher than that among the women in the reference 
Table 3. Characteristics of 4142 pregnancies with live births by likelihood of maternal occupational exposure to endocrine disrupter chemicals (EDC) during pregnancy, Born in Bradford Study, 2007-2012. Bold denotes significance $(P<0.05)$. [Cl=confidence interval; $B M l=b o d y$ mass index; GCSE=general certificate of secondary education; IMD=index of multiple deprivation for Bradford; $P A H=$ polycyclic aromatic hydrocarbons; $\mathrm{POBW}=$ percentage of optimal birth weight; $\mathrm{RR}=$ risk ratio; $\mathrm{SGA}=$ small-for-gestational age.]

\begin{tabular}{|c|c|c|c|c|c|c|c|c|c|c|}
\hline \multirow[t]{2}{*}{ Characteristics $(\mathrm{N}=4142)$} & \multicolumn{2}{|c|}{$\begin{array}{l}\text { Unlikely EDC } \\
\text { exposure }\end{array}$} & \multicolumn{2}{|c|}{$\begin{array}{l}\text { Possible/ probable } \\
\text { EDC exposure }\end{array}$} & \multirow[b]{2}{*}{$\mathrm{Chi}^{2}$} & \multirow[b]{2}{*}{$\operatorname{Pr}$} & \multicolumn{3}{|c|}{$\begin{array}{c}\text { Univariate and } \\
\text { multivariate analyses }\end{array}$} & \multirow[t]{2}{*}{ P-value } \\
\hline & $\mathrm{N}$ & $\%$ & $\mathrm{~N}$ & $\%$ & & & crude RR $(95 \% \mathrm{Cl})$ & P-value & $\mathrm{RR}_{\mathrm{adj}}(95 \% \mathrm{Cl})^{\text {a }}$ & \\
\hline \multicolumn{11}{|l|}{ Exposure to EDC } \\
\hline Any exposure (total) & 3777 & 92.66 & 299 & 7.34 & & & & & & \\
\hline РАH & 4012 & 98.43 & 64 & 1.57 & & & & & & \\
\hline Polychloride organic compounds & 4072 & 99.9 & 4 & 0.1 & & & & & & \\
\hline Pesticides & 4069 & 99.83 & 7 & 0.17 & & & & & & \\
\hline Phthalates & 4000 & 98.14 & 76 & 1.86 & & & & & & \\
\hline Organic solvents & 3895 & 95.56 & 181 & 4.44 & & & & & & \\
\hline Bisphenol A & 4076 & 98.62 & 0 & 0 & & & & & & \\
\hline Alkylphenolics & 3917 & 96.1 & 159 & 3.9 & & & & & & \\
\hline Flame retardants & 4074 & 99.95 & 2 & 0.05 & & & & & & \\
\hline Metals & 4028 & 98.82 & 48 & 1.18 & & & & & & \\
\hline Miscellaneous & 4006 & 98.34 & 70 & 1.72 & & & & & & \\
\hline \multicolumn{11}{|l|}{ Occupational group (mother) b } \\
\hline Managers/seniors & 272 & 7.2 & 8 & 2.68 & \multirow{9}{*}{489.4} & \multirow{9}{*}{$<0.00$} & 1.00 & & 1.00 & \\
\hline Professionals & 435 & 11.52 & 3 & 1 & & & 1.00 & & 1.00 & \\
\hline Associated professionals & 715 & 18.93 & 0 & 0 & & & 1.00 & & 1.00 & \\
\hline Administration/secretarial & 535 & 14.16 & 0 & 0 & & & 1.00 & & 1.00 & \\
\hline Skilled trades & 646 & 17.1 & 30 & 10.03 & & & $7.94(4.00-15.76)$ & $<0.00$ & $12.08(4.95-29.43)$ & $<0.00$ \\
\hline Personal service & 34 & 0.9 & 11 & 3.68 & & & $43.73(20.01-95.58)$ & $<0.00$ & $52.92(19.19-145.92)$ & $<0.00$ \\
\hline Customer service & 716 & 18.96 & 99 & 33.11 & & & $21.73(11.71-40.30)$ & $<0.00$ & $25.10(10.50-60.02)$ & $<0.00$ \\
\hline Machine operatives & 29 & 0.77 & 17 & 5.69 & & & $66.11(32.83-133.13)$ & $<0.00$ & $80.40(31.08-207.98)$ & $<0.00$ \\
\hline Elementary occupation & 395 & 10.46 & 131 & 43.81 & & & $44.55(24.26-81.82)$ & $<0.00$ & $56.40(24.01-132.51)$ & $<0.00$ \\
\hline \multicolumn{11}{|l|}{ Work type } \\
\hline Most time/sitting & 1593 & 49.77 & 48 & 10.05 & \multirow{3}{*}{91.18} & \multirow{3}{*}{$<0.00$} & 1.00 & & 1.00 & \\
\hline Most time/standing & 1229 & 38.39 & 147 & 58.33 & & & $3.65(2.65-5.01)$ & $<0.00$ & $1.83(1.28-2.60)$ & $<0.00$ \\
\hline Physical effort & 379 & 11.84 & 57 & 22.62 & & & $4.47(3.08-6.46)$ & $<0.00$ & $1.64(1.10-2.45)$ & $<0.00$ \\
\hline \multicolumn{11}{|l|}{ Ethnic origin } \\
\hline British Caucasian & 2214 & 58.62 & 224 & 74.92 & \multirow{3}{*}{46.18} & & 1.00 & & 1.00 & \\
\hline South Asian & 1139 & 30.16 & 35 & 11.71 & & $<0.00$ & $0.32(0.22-0.46)$ & $<0.00$ & $0.35(0.22-0.56)$ & $<0.00$ \\
\hline Other & 424 & 11.23 & 40 & 13.38 & & & $0.94(0.68-1.29)$ & $<0.69$ & $0.65(0.44-0.97)$ & $<0.03$ \\
\hline Age (years) & & & & & & & & & & \\
\hline $20-34$ & 3089 & 81.78 & 242 & 80.94 & & & 1.00 & & 1.00 & \\
\hline$>34$ & 553 & 14.64 & 36 & 12.04 & 9.93 & $<0.00$ & $0.84(0.59-1.18)$ & $<0.32$ & $1.04(0.72-1.52)$ & $<0.87$ \\
\hline$<20$ & 135 & 3.57 & 21 & 7.02 & & & $1.85(1.22-2.81)$ & $<0.00$ & $0.94(0.61-1.44)$ & $<0.78$ \\
\hline Education (mother) & & & & & & & & & & \\
\hline$<5$ GCSE equivalents & 262 & 6.95 & 50 & 16.78 & & & 1.00 & & 1.00 & \\
\hline 5 GCSE equivalents & 1005 & 26.67 & 102 & 34.23 & & & $0.57(0.42-0.78)$ & $<0.00$ & $0.79(0.57-1.10)$ & $<0.14$ \\
\hline A-level equivalent ${ }^{c}$ & 763 & 20.25 & 46 & 15.44 & 69.76 & $<0.00$ & $0.35(0.24-0.52)$ & $<0.00$ & $0.58(0.39-0.86)$ & $<0.00$ \\
\hline$>$ A level & 1378 & 36.57 & 60 & 20.13 & & & $0.26(0.18-0.37)$ & $<0.00$ & $0.77(0.50-1.20)$ & $<0.25$ \\
\hline Other & 296 & 7.86 & 31 & 10.4 & & & $0.59(0.39-0.90)$ & $<0.01$ & $1.04(0.66-1.62)$ & $<0.89$ \\
\hline Unknown & 64 & 1.7 & 9 & 3.02 & & & $0.77(0.39-1.49)$ & $<0.43$ & $0.72(0.33-1.59)$ & $<0.42$ \\
\hline Smoking (cigarettes) & & & & & & & & & & \\
\hline 0 & 3222 & 85.37 & 215 & 71.91 & & & 1.00 & & 1.00 & \\
\hline 1-5 per day & 244 & 6.47 & 37 & 12.37 & 38.13 & $<0.00$ & $2.10(1.51-2.91)$ & $<0.00$ & $1.33(0.96-1.84)$ & $<0.08$ \\
\hline$>5$ per day & 308 & 8.16 & 47 & 15.72 & & & $2.12(1.57-2.84)$ & $<0.00$ & $1.01(0.73-1.40)$ & $<0.94$ \\
\hline Alcohol & & & & & & & & & & \\
\hline No/occasionally & 2938 & 72.2 & 197 & 65.89 & & & 1.00 & & 1.00 & \\
\hline Yes & 1131 & 27.8 & 102 & 34.11 & 6.41 & $<0.01$ & $1.34(1.06-1.69)$ & $<0.01$ & $1.10(0.86-1.42)$ & $<0.39$ \\
\hline $\mathrm{BMI}$ & & & & & & & & & & \\
\hline Normal & 986 & 26.84 & 85 & 29.31 & & & 1.00 & & 1.00 & \\
\hline Overweight & 1462 & 39.8 & 113 & 38.97 & 1.69 & $<0.63$ & $0.90(0.68-1.18)$ & $<0.46$ & $1.00(0.75-1.33)$ & $<0.99$ \\
\hline Obese & 1220 & 33.22 & 91 & 31.38 & & & $0.87(0.66-1.16)$ & $<0.35$ & $0.98(0.73-1.32)$ & $<0.91$ \\
\hline Underweight & 5 & 0.14 & $<5 \mathrm{~g}$ & 0.34 & & & $2.10(0.34-12.71)$ & $<0.41$ & $4.56(2.17-9.56)$ & $<0.00$ \\
\hline
\end{tabular}

group. Exposures to ALP was statistically non-significantly associated with increased risk of $\mathrm{POBW}<85$. No association was found between $\mathrm{POBW}<85$ and exposures to PAH, organic solvents, and metals. Exposure to the miscellaneous category had a protective effect.

The most frequently occurring occupations associated with exposure to pesticides with significant adverse effects on fetal growth were veterinary nurses, veterinary assistants, and horticultural trades. The main pesticides encountered were carbamates, organophosphates and pyrethroids. The most prevalent occupations associated with exposure to phthalates with significant adverse effects on fetal growth were hairdressers, beauticians and related occupations and printing machine minders. 
Table 3. continued

\begin{tabular}{|c|c|c|c|c|c|c|c|c|c|c|}
\hline \multirow[t]{2}{*}{ Characteristics $(\mathrm{N}=4142)$} & \multicolumn{2}{|c|}{$\begin{array}{l}\text { Unlikely EDC } \\
\text { exposure }\end{array}$} & \multicolumn{2}{|c|}{$\begin{array}{l}\text { Possible/ probable } \\
\text { EDC exposure }\end{array}$} & \multirow[b]{2}{*}{$\mathrm{Chi}^{2}$} & \multirow[b]{2}{*}{$\mathrm{Pr}$} & \multicolumn{3}{|c|}{$\begin{array}{c}\text { Univariate and } \\
\text { multivariate analyses }\end{array}$} & \multirow[t]{2}{*}{ P-value } \\
\hline & $\mathrm{N}$ & $\%$ & $\mathrm{~N}$ & $\%$ & & & crude RR $(95 \% \mathrm{Cl})$ & P-value & $\mathrm{RR}_{\mathrm{adj}}(95 \% \mathrm{Cl})^{\mathrm{b}}$ & \\
\hline \multicolumn{11}{|l|}{ Parity } \\
\hline First child & 1946 & 53.45 & 161 & 55.52 & & & 1.00 & & 1.00 & \\
\hline Second child \& higher & 1695 & 46.55 & 129 & 44.48 & 0.46 & $<0.49$ & $0.92(0.74-1.15)$ & $<0.49$ & $0.87(0.68-1.10)$ & ) $<0.28$ \\
\hline \multicolumn{11}{|l|}{ Marital Status } \\
\hline Married/remarried & 2334 & 61.86 & 116 & 38.8 & \multirow{4}{*}{63.35} & \multirow{3}{*}{$<0.00$} & 1.00 & & 1.00 & \\
\hline Single & 1336 & 35.41 & 174 & 58.19 & & & $2.43(1.94-3.05)$ & $<0.00$ & $1.22(0.91-1.63)$ & ) $<0.17$ \\
\hline Separated/divorced/ widowed & 103 & 2.73 & 9 & 3.01 & & & $1.69(0.88-3.25)$ & $<0.11$ & $0.96(0.47-1.92)$ & ) $<0.92$ \\
\hline \multicolumn{10}{|l|}{ IMD 2010 score BF } & \\
\hline 1 (most deprived) & 857 & 23.21 & 70 & 23.73 & \multirow{5}{*}{6.9} & \multirow{5}{*}{$<0.14$} & 1.00 & & 1.00 & \\
\hline 2 & 861 & 23.32 & 77 & 26.1 & & & $1.08(0.79-1.48)$ & $<0.59$ & $1.17(0.85-1.60)$ & $<0.29$ \\
\hline 3 & 907 & 24.57 & 82 & 27.8 & & & $1.09(0.80-1.49)$ & $<0.55$ & $1.38(1.00-1.90)$ & $<0.04$ \\
\hline 4 & 815 & 22.07 & 47 & 15.93 & & & $0.72(0.50-1.03)$ & $<0.07$ & $1.05(0.72-1.54)$ & $<0.74$ \\
\hline 5 (least deprived) & 252 & 6.83 & 19 & 6.44 & & & $0.92(0.56-1.51)$ & $<0.76$ & $1.79(1.06-3.05)$ & $<0.02$ \\
\hline
\end{tabular}

a Adjusted for all variables in the table and job hours during pregnancy $(<35, \geq 35)$, alcohol consumption (drank alcohol during pregnancy (yes or no/occasionally), marital status (married/remarried, single, separated/divorced, widowed), parity (first child, second child \& higher), gestational diabetes (no, yes), preeclampsia (no, yes), pre-exciting hypertension (no, yes), other tobacco (no, yes), and drugs during pregnancy (no, yes).

${ }^{\mathrm{D}}$ The first four occupational groups combined and used as the reference group in univariate and multivariate analyses.

${ }^{\mathrm{C}} \mathrm{A}$-Level- A-C equates to Level 2 attainment defined by the 2011 revision of the International Standard Classification of Education; $\geq 2$ advanced levels or equivalent qualifications equate to Level 3 educational attainment.

The phthalates most often encountered were DEHP, BBP, DBP, and DEP. The most prevalent occupations associated with exposure to ALP with significant effect on fetal growth were domestic cleaners, hairdressers and beauticians. The ALP most often encountered were alklylphenols and alkylphenolic ethoxylates.

Table 3 shows the distribution of pregnancy characteristics of 4142 stratified by likelihood of maternal occupational exposure to EDC during pregnancy. Almost $7.5 \%$ of the study cohort were classified as possibly or probably exposed to $\geq 1$ of 10 classes of EDC. The most common encountered exposures were to organic solvents $(4.5 \%)$ and ALP (4\%) phthalates (1.9\%), PAH (1.6\%), metals (1.2\%), and miscellaneous $(1.7 \%)$. In general, women who were more likely to be exposed to EDC worked in skilled trades, personal service, elementary occupations, or as machine operators. In addition, their work involved prolonged standing or physical effort and they were more likely to be Causcasian British and less educated.

\section{Discussion}

This study provides evidence that maternal occupational exposure during pregnancy to estimated concentrations of EDC - as classified by application of a JEM - is associated with significantly increased risk of impaired fetal growth. In particular, mothers exposed to pesticides were three to five times more likely to have an infant with suboptimal fetal growth as measured by $\mathrm{POBW}<85$ and SGA respectively, and mothers exposed to phthalates were about three times more likely to have a baby with inadequate fetal growth measured by $\mathrm{POBW}<85$. Maternal exposure to ALP was associated with a nonsignificant but increased risk of inadequate fetal growth as measured by SGA and $\mathrm{POBW}<85$.

This study also demonstrated disproportionate exposure to EDC with personal risk factors in women. In general, women who were exposed to EDC were more likely to be Caucasian British, less educated, done work involving prolonged standing or physical effort and worked as skilled trades, personal service, machine operators and elementary occupations.

The study has several strengths primarily due to the large amount and detail of data available. The prospective design minimises recall bias, and selection bias was minimised by the $80 \%$ participation rate to the mid-pregnancy. Detailed information was collected about individual maternal characteristics and information obtained on chemical exposures through JEM, which enabled adjustment for potential confounders including adjustment for exposures to individual EDC in order to minimise the effect of possible confounding. The classification of EDC exposures was assessed independently and prior to knowledge of the outcomes by a recently updated JEM developed specifically to assess the association between occupational exposures to EDC and birth outcomes, thus information bias was largely eliminated. We were able to evaluate the effect of several EDC exposures on two different criteria for inadequate fetal growth.

In this study, we used POBW $<85$ as an indicator of inadequate intrauterine growth that is less dependent on the health of the reference population or the quality of their morphometric data than is percentile position on a birth weight distribution. The method uses optimal 
rather than expected growth as the standard and reports the ratio of the observed birth dimension to the optimal birth dimension rather than as being above or below a specified position of the population distribution of that dimension, avoiding the problems inherent in the use of percentile position.

The availability of job titles, the detailed information on work tasks or activities routinely performed, type of business and information about when the mother worked during her pregnancy has reduced non-differential misclassification of exposure and enabled a more accurate assessment of occupational exposures compared with studies with access only to job titles.

We validated the job title coding to get a more accurate code for each job title, so the potential for observer bias in the coding of occupational title status was minimised, improving the reliability and validity of the coded job titles. The risk for each category of EDC was estimated, rather than several EDC categories combined, allowing any differences between categories to be observed. Finally, JEM-based assessments of risk of exposures to chemical agents are more reliable than self-reported assessments (17).

A limitation was that individual exposures were not measured. However, Vandenberg et al (44), has reviewed the dose-response between endocrine disrupters and various health outcomes and the possibility of non-monotonic dose-responses. As stated by Vandenberg: "the endocrine system evolved to function when unbound physiologically active ligand (hormones) are present at extremely low doses", "EDCs that mimic natural hormones have been proposed to follow the same rules and therefore have biological effects at low doses" (44, page 8). Another limitation is that the cells of JEM represent exposure probabilities, which are only a crude measure of exposure, so it needs to be interpreted with caution. Furthermore, the JEM does not consider specific chemicals but only broad groups thereof, and the mechanisms of action can vary between specific chemicals in a group (34). In this study, we reported the specific chemicals identified within each broad group of pesticides, phthalates and alkylphenolic compounds, but it was not possible to distinguish the role of each specific chemicals in their broad groups in the observed lower fetal growth rate. There is also a possibility of overlap between the categories of phthalates and alkyphenolic compounds among exposed mothers, so it was not possible to separate the specific role of each of these chemicals in inadequate fetal growth rate. Finally, too few mothers were exposed to some of EDC such as polychlorinated organic compounds, bisphenol $\mathrm{A}$, and flame-retardants to allow evaluation of their associations with our outcomes. Exposure to the miscellaneous category had a protective effect, but due to small number of exposed people, the CI was very large, $(95 \%$
CI $0.05-0.60$ ) so the results should be interpreted with caution. Large CI were also observed in the results of occupational groups in table 3 . There might be a risk of type 2 errors due to small samples of those exposed to some of EDC. However, for other EDC our results are compatible with those of previous similar studies (4, $16,17)$, enhancing their credibility as well as our own.

This study introduces another approach (fetal growth measured by percentage of optimal birth weight) for defining adequacy of growth in assessing the effects of chemical exposures compared to other published studies. To our knowledge, this is the first study to show that the risk of inadequate fetal growth as measured by POBW $<85$ was significantly elevated following possible or probable maternal occupational exposures to one or more classes of EDC, particularly pesticides and phthalates. A recent study in the Generation $\mathrm{R}$ cohort using the same JEM found that occupational exposures to pesticides and phthalates during pregnancy were significantly associated with reduced placental weight and fetal length as estimated by ultrasounds and reduced fetal weight following mother's exposures to phthalates and PAHs (16). Another study from Generation $\mathrm{R}$ cohort using the same JEM concluded that maternal occupational exposure to phthalates and pesticides was associated with adverse effects on fertility and pregnancy outcomes (17). A meta-analysis from a European large-scale prospective study using the same JEM also suggests that pregnant women classified as exposed to multiple EDC, including pesticides and phthalates, were at significantly higher risk of term low birth weight newborns in cohorts throughout Europe (4). Our finding in regard to non-significant association between $\mathrm{POBW}<85$ as a measure of fetal growth and maternal exposure to ALP is in line with a recent study in the Generation R cohort using the same JEM in assessing occupational exposure to chemicals and fetal growth as measured by reduced fetal weight estimated from ultrasound-fetometry (16). Our finding about a significant association between maternal exposure to pesticides and SGA is supported by several studies (45-47). However, epidemiological studies on the effect of exposures to endocrine disrupters on pregnancy outcomes are not always consistent, warranting further research into this important topic. For example, the affected occupations associated with exposures to pesticides in this study were those classified as veterinary nurses and horticultural trades and, to phthalates, hairdressers, beauticians, and printing machine minders. The findings in the present study concerning exposure to pesticides and phthalates in hairdressers and agricultural activities and having infants with inadequate fetal growth is in accordance with previous findings $(16,17,48-50)$. However, there were some studies in agricultural activities and among hairdressers that show conflicting results (51-54). 
There is limited research evaluating the occupational and personal characteristics of women associated with occupational exposures to EDC. Evaluation of the influence of both occupational and personal risk factors (smoking, alcohol consumption, age, marital status, ethnic origin, education, BMI, socio-economic status) would help to improve our understanding of health hazards and develop a comprehensive preventive approach to achieve a longer, healthy working life. Unequal exposure to occupational exposure acting as EDC is an under-recognized risk factor that may play an important role in deriving the higher rates of adverse pregnancy outcomes among those affected populations.

Human development is most vulnerable to toxic substances and endocrine disruption in the early embryonic period. The restricted fetal growth associated with exposure to pesticides and phthalates during pregnancy is an important public health concern because restricted fetal growth is linked to adverse health later in life such as coronary heart disease, stroke, type 2 diabetes, and hypertension (55). It is therefore important to identify occupation-related risk factors for adverse pregnancy outcomes. Further larger studies are needed to confirm these findings and identify potential targets for prevention. Until then, precautionary prevention and control management of risks to health and safety at the workplace are recommended. In general, the precautions to be taken for the protection of the reproductive health of both women and men will not differ from the safeguarding of all workers. A national priority of supporting research on occupational causes of adverse reproductive outcomes recommended.

\section{Concluding remarks}

Consistent with the results of other studies, this prospective birth cohort study provides evidence that occupational exposure to pesticides and phthalates may play a role in the etiology of inadequate fetal growth and SGA infants.

\section{Acknowledgements}

The Born in Bradford Study is only possible because of the enthusiasm and commitment of participating children and parents. We are grateful to all the participants, health professionals and researchers who have made the study happen. The authors also would like to acknowledge the Sir Walter Murdoch School of Public Policy \& International Affairs for infrastructure support that enabled Dr Shirangi to complete this study.

\section{Funding}

The National Health and Medical Research Council of Australia supported this work, (Grant ID: 463908 to Dr Adeleh Shirangi for a Sidney Sax Fellowship). Funding Data Registry: researchdata.ands.org.au/dr-adelehshirangi/523275.

Professor John Wright and Dr Rosemary McEachan received funding from the National Institute for Health Research's Yorkshire and Humber Applied Research Collaboration (NIHR200166) and A Wellcome infrastructure grant (WT101597MA). The views expressed in this publication are those of the authors and not necessarily those of the National Institute for Health Research or the Department of Health and Social Care.

\section{Conflict of interest}

The authors have no conflicts of interest to declare.

\section{References}

1. Bergman A, Heindel JJ, Joblin S, Kidd KA, Zoeller RT State of the Science of Endocrine Disrupting Chemicals. WHO/UNEP (World Health Organization/ United Nations Environment Program). 2012;1-261.

2. Sharpe RM, Irvine DS. How strong is the evidence of a link between environmental chemicals and adverse effects on human reproductive health? BMJ $2004 \mathrm{Feb} ; 328(7437)$ :44751. https://doi.org/10.1136/bmj.328.7437.447.

3. Burdorf A, Nieuwenhuijsen MJ. Endocrine disrupting chemicals and human reproduction: fact or fiction? Ann Occup Hyg 1999 Oct;43(7):435-7.

4. Birks L, Casas M, Garcia AM, Alexander J, Barros H, Bergström A et al.; Regina Gražulevičienè. Occupational Exposure to Endocrine-Disrupting Chemicals and Birth Weight and Length of Gestation: A European MetaAnalysis. Environ Health Perspect 2016 Nov; 124(11):178593. https://doi.org/10.1289/EHP208.

5. Burdorf A, Figà-Talamanca I, Jensen TK, Thulstrup AM. Effects of occupational exposure on the reproductive system: core evidence and practical implications. Occup Med (Lond) 2006 Dec;56(8):516-20. https://doi.org/10.1093/occmed/ kq1113.

6. Verick S. Female labor force participation in developing countries. IZA World of Labor 2014: 87, wol.iza.org

7. Shirangi A, Fritschi L, Holman CD. Maternal occupational exposures and risk of spontaneous abortion in veterinary practice. Occup Environ Med 2008 Nov;65(11):719-25. https://doi.org/10.1136/oem.2007.035246.

8. Shirangi A, Fritschi L, Holman CD, Bower C. Birth defects in offspring of female veterinarians. J Occup Environ Med 2009 May;51(5):525-33. https://doi.org/10.1097/ JOM.0b013e3181a01af3. 
9. Vrijheid M, Armstrong B, Dolk H, van Tongeren M, Botting B. Risk of hypospadias in relation to maternal occupational exposure to potential endocrine disrupting chemicals. Occup Environ Med 2003 Aug;60(8):543-50. https://doi. org/10.1136/oem.60.8.543.

10. Ormond G, Nieuwenhuijsen MJ, Nelson P, Toledano MB, Iszatt $\mathrm{N}$, Geneletti $\mathrm{S}$ et al. Endocrine disruptors in the workplace, hair spray, folate supplementation, and risk of hypospadias: case-control study. Environ Health Perspect 2009 Feb;117(2):303-7. https://doi.org/10.1289/ehp.11933.

11. Shirangi A, Bower C, Holman CD, Preen DB, Bruce N. A study of handling cytotoxic drugs and risk of birth defects in offspring of female veterinarians. Int J Environ Res Public Health 2014 Jun;11(6):6216-30. https://doi.org/10.3390/ ijerph110606216.

12. Spinder N, Prins JR, Bergman JE, Smidt N, Kromhout H, Boezen HM et al. Congenital anomalies in the offspring of occupationally exposed mothers: a systematic review and meta-analysis of studies using expert assessment for occupational exposures. Hum Reprod 2019 May;34(5):90319. https://doi.org/10.1093/humrep/dez033.

13. Nurminen T. Maternal pesticide exposure and pregnancy outcome. J Occup Environ Med 1995 Aug;37(8):935-40. https://doi.org/10.1097/00043764-199508000-00008.

14. Govarts E, Iszatt N, Trnovec T, de Cock M, Eggesbo M, Palkovicova Murinova L, et al. Prenatal exposure to endocrine disrupting chemicals and risk of being born small for gestational age: Pooled analysis of seven European birth cohorts. Environment international. 2018;115(267-78). https://doi.org/10.1016/j.envint.2018.03.017.

15. Snijder CA, Heederik D, Pierik FH, Hofman A, Jaddoe VW, Koch HM et al. Fetal growth and prenatal exposure to bisphenol A: the generation R study. Environ Health Perspect 2013 Mar;121(3):393-8. https://doi.org/10.1289/ ehp. 1205296.

16. Snijder CA, Roeleveld N, Te Velde E, Steegers EA, Raat H, Hofman A et al. Occupational exposure to chemicals and fetal growth: the Generation R Study. Hum Reprod 2012 Mar;27(3):910-20. https://doi.org/10.1093/humrep/der437.

17. Burdorf A, Brand T, Jaddoe VW, Hofman A, Mackenbach JP, Steegers EA. The effects of work-related maternal risk factors on time to pregnancy, preterm birth and birth weight: the Generation R Study. Occup Environ Med 2011 Mar;68(3):197-204. https://doi.org/10.1136/ oem.2009.046516.

18. Shirangi A, Fritschi L, Holman CD. Associations of unscavenged anesthetic gases and long working hours with preterm delivery in female veterinarians. Obstet Gynecol 2009 May;113(5):1008-17. https://doi.org/10.1097/ AOG.0b013e31819fe996.

19. Barker DJ, Martyn CN, Osmond C, Hales CN, Fall CH. Growth in utero and serum cholesterol concentrations in adult life. BMJ 1993 Dec;307(6918):1524-7. https://doi. org/10.1136/bmj.307.6918.1524.

20. Barker DJ, Osmond C, Simmonds SJ, Wield GA. The relation of small head circumference and thinness at birth to death from cardiovascular disease in adult life. BMJ 1993 Feb;306(6875):422-6. https://doi.org/10.1136/ bmj.306.6875.422.

21. McCormick MC. The contribution of low birth weight to infant mortality and childhood morbidity. N Engl J Med 1985 Jan;312(2):82-90. https://doi.org/10.1056/ NEJM198501103120204.

22. Saigal S, Doyle LW. An overview of mortality and sequelae of preterm birth from infancy to adulthood. Lancet 2008 Jan;371(9608):261-9. https://doi.org/10.1016/S01406736(08)60136-1.

23. Leonard H, Nassar N, Bourke J, Blair E, Mulroy S, de Klerk $\mathrm{N}$ et al. Relation between intrauterine growth and subsequent intellectual disability in a ten-year population cohort of children in Western Australia. Am J Epidemiol 2008 Jan;167(1):103-11. https://doi.org/10.1093/aje/kwm245.

24. Miranda ML, Maxson P, Edwards S. Environmental contributions to disparities in pregnancy outcomes. Epidemiol Rev. 2009;31:67-83.

25. Langridge AT, Nassar N, Li J, Stanley FJ. Social and racial inequalities in preterm births in Western Australia, 1984 to 2006. Paediatr Perinat Epidemiol. 24(4):352-62.

26. Shirangi A, Nieuwenhuijsen M, Vienneau D, Holman CD Living near agricultural pesticide applications and the risk of adverse reproductive outcomes: a review of the literature. Paediatr Perinat Epidemiol 2011 Mar;25(2):172-91. https:// doi.org/10.1111/j.1365-3016.2010.01165.x.

27. Kozuki N, Lee AC, Silveira MF, Sania A, Vogel JP, Adair L, et al. The associations of parity and maternal age with smallfor-gestational-age, preterm, and neonatal and infant mortality: a meta-analysis. BMC public health. 2013;13 Suppl 3(S2).

28. Dranitsaris G, Johnston M, Poirier S, Schueller T, Milliken $\mathrm{D}$, Green $\mathrm{E}$ et al. Are health care providers who work with cancer drugs at an increased risk for toxic events? A systematic review and meta-analysis of the literature. J Oncol Pharm Pract 2005 Jun;11(2):69-78. https://doi. org/10.1191/1078155205jp155oa.

29. Wright J, Small N, Raynor P, Tuffnell D, Bhopal R, Cameron $\mathrm{N}$ et al.; Born in Bradford Scientific Collaborators Group. Cohort Profile: the Born in Bradford multi-ethnic family cohort study. Int J Epidemiol 2013 Aug;42(4):978-91. https://doi.org/10.1093/ije/dys112.

30. Sheridan E, Wright J, Small N, Corry PC, Oddie S, Whibley $\mathrm{C}$ et al. Risk factors for congenital anomaly in a multiethnic birth cohort: an analysis of the Born in Bradford study. Lancet 2013 Oct;382(9901):1350-9. https://doi. org/10.1016/S0140-6736(13)61132-0.

31. Classification SO. Office of National Statistics. 2000, Volume 1: Structure and descriptions of unit groups. Available from: http://www.ons.gov.uk/about-statistics/classifications/ archived/SOC2000/index.html.

32. Cascot: Computer Assisted Structured Coding Tool. Warwick Institute for Employment Research. Available from: https:// warwick.ac.uk/fac/soc/ier/. 
33. Van Tongeren M, Nieuwenhuijsen MJ, Gardiner K, Armstrong B, Vrijheid M, Dolk H et al. A job-exposure matrix for potential endocrine-disrupting chemicals developed for a study into the association between maternal occupational exposure and hypospadias. Ann Occup Hyg 2002 Jul;46(5):465-77.

34. Brouwers MM, van Tongeren M, Hirst AA, Bretveld RW, Roeleveld N. Occupational exposure to potential endocrine disruptors: further development of a job exposure matrix. Occup Environ Med 2009 Sep;66(9):607-14. https://doi. org/10.1136/oem.2008.042184.

35. Norris T, Johnson W, Farrar D, Tuffnell D, Wright J, Cameron N. Small-for-gestational age and large-forgestational age thresholds to predict infants at risk of adverse delivery and neonatal outcomes: are current charts adequate? An observational study from the Born in Bradford cohort. BMJ Open 2015 Mar;5(3):e006743. https://doi. org/10.1136/bmjopen-2014-006743.

36. Stacey T, Prady S, Haith-Cooper M, Downe S, Simpson N, Pickett K. Ethno-Specific Risk Factors for Adverse Pregnancy Outcomes: Findings from the Born in Bradford Cohort Study. Matern Child Health J 2016 Jul;20(7):1394404. https://doi.org/10.1007/s10995-016-1936-x.

37. Gardosi J, Chang A, Kalyan B, Sahota D, Symonds EM. Customised antenatal growth charts. Lancet 1992 Feb;339(8788):283-7. https://doi.org/10.1016/01406736(92)91342-6.

38. Blair EM, Liu Y, de Klerk NH, Lawrence DM. Optimal fetal growth for the Caucasian singleton and assessment of appropriateness of fetal growth: an analysis of a total population perinatal database. BMC Pediatr 2005 May;5(1):13. https://doi.org/10.1186/1471-2431-5-13.

39. Pereira G, Blair E, Lawrence D. Validation of a model for optimal birth weight: a prospective study using serial ultrasounds. BMC Pediatr 2012 Jun;12(73):73. https://doi. org/10.1186/1471-2431-12-73.

40. Malacova E, Li J, Blair E, Leonard H, de Klerk N, Stanley F. Association of birth outcomes and maternal, school, and neighborhood characteristics with subsequent numeracy achievement. Am J Epidemiol 2008 Jul;168(1):21-9. https:// doi.org/10.1093/aje/kwn085.

41. Laurvick CL, Milne E, Blair E, de Klerk N, Charles AK, Bower C. Fetal growth and the risk of childhood non-CNS solid tumours in Western Australia. Br J Cancer 2008 Jul;99(1):179-81. https://doi.org/10.1038/sj.bjc.6604424.

42. McNutt LA, Wu C, Xue X, Hafner JP. Estimating the relative risk in cohort studies and clinical trials of common outcomes. Am J Epidemiol 2003 May;157(10):940-3. https://doi.org/10.1093/aje/kwg074.

43. Zou G. A modified poisson regression approach to prospective studies with binary data. Am J Epidemiol 2004 Apr;159(7):702-6. https://doi.org/10.1093/aje/kwh090.
44. Vandenberg LN, Colborn T, Hayes TB, Heindel JJ, Jacobs DR Jr, Lee DH et al. Hormones and endocrine-disrupting chemicals: low-dose effects and nonmonotonic dose responses. Endocr Rev 2012 Jun;33(3):378-455. https://doi. org/10.1210/er.2011-1050.

45. Savitz DA, Whelan EA, Kleckner RC. Self-reported exposure to pesticides and radiation related to pregnancy outcome--results from National Natality and Fetal Mortality Surveys. Public Health Rep 1989 Sep-Oct;104(5):473-7.

46. Zhang J, Cai WW, Lee DJ. Occupational hazards and pregnancy outcomes. Am J Ind Med 1992;21(3):397-408. https://doi.org/10.1002/ajim.4700210312.

47. Seidler A, Raum E, Arabin B, Hellenbrand W, Walter U, Schwartz FW. Maternal occupational exposure to chemical substances and the risk of infants small-for-gestationalage. Am J Ind Med 1999 Jul;36(1):213-22. https://doi. org/10.1002/(SICI) 1097-0274(199907)36:1<213::AIDAJIM30>3.0.CO;2-A.

48. Rylander L, Källén B. Reproductive outcomes among hairdressers. Scand J Work Environ Health 2005 Jun;31(3):212-7. https://doi.org/10.5271/sjweh.871.

49. Wigle DT, Arbuckle TE, Turner MC, Bérubé A, Yang Q, Liu $\mathrm{S}$ et al. Epidemiologic evidence of relationships between reproductive and child health outcomes and environmental chemical contaminants. J Toxicol Environ Health B Crit Rev 2008 May;11(5-6):373-517. https://doi. org/10.1080/10937400801921320.

50. Stillerman KP, Mattison DR, Giudice LC, Woodruff TJ. Environmental exposures and adverse pregnancy outcomes: a review of the science. Reprod Sci 2008 Sep;15(7):631-50. https://doi.org/10.1177/1933719108322436.

51. Petit C, Chevrier C, Durand G, Monfort C, Rouget F, Garlantezec R, et al. Impact on fetal growth of prenatal exposure to pesticides due to agricultural activities: a prospective cohort study in Brittany, France. Environ Health. 2010;9(1):71. https://doi.org/10.1186/1476-069X-9-71.

52. Zhu JL, Hjollund NH, Andersen AM, Olsen J. Occupational exposure to pesticides and pregnancy outcomes in gardeners and farmers: a study within the Danish National Birth Cohort. J Occup Environ Med 2006 Apr;48(4):347-52. https://doi.org/10.1097/01.jom.0000201566.42186.5f.

53. Zhu JL, Vestergaard M, Hjollund NH, Olsen J. Pregnancy outcomes among female hairdressers who participated in the Danish National Birth Cohort. Scand J Work Environ Health 2006 Feb;32(1):61-6. https://doi.org/10.5271/sjweh.977.

54. Axmon A, Rylander L. Birth weight and fetal growth in infants born to female hairdressers and their sisters. Occup Environ Med 2009 Mar;66(3):198-204. https://doi. org/10.1136/oem.2008.039784.

55. Barker DJ, Eriksson JG, Forsén T, Osmond C. Fetal origins of adult disease: strength of effects and biological basis. Int $\mathrm{J}$ Epidemiol 2002 Dec;31(6):1235-9. https://doi.org/10.1093/ ije/31.6.1235.

Received for publication: 17 May 2019 